\title{
Transplantation of tissues and organs ${ }^{1}$
}

In recent years transplantation biology has emerged as a major branch of immunology and one that embraces many interrelated disciplines. The number of papers on the subject has increased so rapidly that the general pathologist may begin to feel he would like some guidance in his reading. To meet this need the College of Pathologists in February of this year held a symposium on the transplantation of tissues and organs, and the 14 papers presented at the meeting are to be published under the aegis of this journal in June, and for the convenience of workers in this field and that of librarians all over the world, the pages will be numbered in sequence with the Journal of Clinical Pathology. At the symposium the advances that have been made in transplantation biology and the problems that remain to be solved were reviewed and discussed.

Of the various ways in which the outcome of organ transplantation might be improved, one of the more promising involves typing and matching donors and recipients. Dr. J. J. van Rood, a pioneer in this field, described the encouraging attempts that are being made to develop highly specific monovalent antisera capable of identifying at least the major transplantation antigens. Although he was very cautious, it seems likely that within a few years it will be possible to type individuals with respect to their transplantation antigens, with much the same degree of accuracy as they can at present be typed with respect to their red blood cell groups. However, as became clear from Dr. J. R. Batchelor's review, at present our knowledge of the genetics of tissue incompatibility is still rudimentary. We do not know how many histocompatibility group systems there are in man and we still are not sure how many of them determine strong antigens.

Although the use of tissue typing in conjunction with current immunosuppressive drug therapy will undoubtedly lead to improved and more consistent results in clinical organ transplantation, application of this combination is unlikely to provide an entirely satisfactory solution to the problem. Ideally a treatment is required that will make the host completely and specifically tolerant of the foreign antigens of the graft, avoiding the need for continued drug treatment. Since 1953 it has been known that inoculation of very young animals with suspensions of living foreign cells makes these animals incapable of reacting against subsequent grafts from the donor of the injected cells. Immunological tolerance can also be induced in adult animals and Dr. N. A. Mitchison in his paper emphasized that there are two different dosage thresholds, one verylow, for achieving tolerance rather than immunization. Unfortunately this approach is at present impractical in man. It must await the day when highly purified transplantation antigens, prepared from different, typed, human cell lines propagated in vitro, are available and can be used to induce tolerance of appropriately matched organ transplants.

At present the most commonly used immunosuppressive agents in clinical renal transplantation are azathioprine and prednisone. The mechanism of action of these, and other drugs that are known to modify the response to foreign grafts, is far from clear and was discussed in detail by Dr. M. C. Berenbaum at the symposium.

Recently a powerful immunosuppressive agent, antilymphocytic serum, has been arousing a great deal of interest. This is the name given to an antiserum raised in members of one species by the injection of lymphoid cells taken from members of another species. Professor M. F. A. Woodruff, who has been largely responsible for giving the transplanter this new weapon, described how this substance can prevent rejection of grafts and even arrest rejection when it has commenced. Dogs treated with horse anti-dog lymphocytic serum have retained renal and hepatic grafts from other unrelated dogs for long periods. Preliminary trials of antilymphocytic serum in patients with renal transplants suggest the material is not toxic and that it may be very effective in combination with azathioprine. There is still no general agreement on the mode of action of antilymphocytic serum.

${ }^{1}$ Full details of prices and how to obtain this publication are set out on page 307. 
One possibility is that it brings about a generalized 'sterile activation' of the recipient's lymphoid cells which supplants all other immunological commitments.

Another group of substances of potentially great value in clinical practice are the ribo-nuclease complexes. Dr. J. F. Mowbray, who has been responsible for the discovery of their immunosuppressive properties, described how in mice the rejection of skin grafts from other mice and even from rats can be prevented.

In considering ways of modifying the rejection of foreign grafts is should not be forgotten that we still do not really understand the mechanism by which the host recognizes and then reacts against a transplant. A lot of time was devoted to these topics at the symposium. Many investigators believe that the agents of recognition and destruction are lymphocytes. The evidence supporting this concept was critically examined by Dr. J. L. Turk. However, macrophages are present in many of the events associated with transplantation immunity and may, for example, cooperate with sensitized lymphocytes in the rejection of grafts. This and other possibilities were discussed by Dr. D. C. Dumonde. The part played by circulating antibody in the rejection of grafts is still not clear and was fully reviewed by Dr. G. Möller, who also talked about allogeneic inhibition, a phenomenon which suggests that there may be a further surveillance mechanism independent of the more widely studied conventional immunological defences.

In recent times the crucial importance of the thymus in the development of immunological activity has been realized, and in the symposium Dr. Delphine Parrott discussed the evidence that some lymphoid tissue is thymus dependent and concerned with graft rejection, while the remainder is responsible for the production of circulating antibodies.

In the latter part of the symposium the transplantation of some individual organs and tissues was considered. Professor K. A. Porter emphasized the vascular nature of the damage to renal transplants during rejection and he distinguished between the early cell-mediated episodes and the later episodes in which circulating antibody may play an important role. Mr. T. Gibson discussed cartilage grafts and pointed out that their success was due to the antigenically inert martix which imposes a physical barrier between the host cells and chondrocytes. Dr. J. F. Loutit authoritatively considered bone marrow transplantation, in particular the nature of haemopoietic stem cells. Finally, bone grafts, which serve as a framework to be replaced by the recipient's tissues, were discussed by Mr. J. Chalmers. 